\title{
CHARACTERISTIC CLASSES AND REPRESENTATIONS OF DISCRETE SUBGROUPS OF LIE GROUPS
}

\author{
BY WILLIAM M. GOLDMAN
}

A volume invariant is used to characterize those representations of a countable group into a connected semisimple Lie group $G$ which are injective and whose image is a discrete cocompact subgroup of $G$. Let $\pi$ be a discrete cocompact subgroup of $G$ and consider the analytic variety $\operatorname{Hom}(\pi, G)$ consisting of homomorphisms $\phi: \pi \rightarrow G$. Denote by $K$ a maximal compact subgroup of $G$ and $X=K \backslash G$ the associated symmetric space. Let $M$ be the orbit space $X / \pi$.

(For convenience we shall henceforth assume that $\pi$ is torsionfree: by Selberg's lemma [12] this may be accomplished by replacing $\pi$ by a subgroup of finite index. This insures that $M$ is a compact smooth manifold having $\pi$ as its fundamental group. The case when $\pi$ has torsion follows from the torsionfree case with minor modifications but these modifications need not concern us here.)

To every representation $\phi \in \operatorname{Hom}(\pi, G)$ we associate a foliated bundle $E_{\phi}$ over $M$ with fibre $X$ and structure group $G$ (see e.g. [6]). If $\omega$ is a closed $G$ invariant differential $k$-form on $X$ then we may spread $\omega$ over the fibres of $E_{\phi}$ (copies of $X$ ) to obtain a closed $k$-form $\omega_{\phi}$ on $E_{\phi}$. We define $\omega(\phi)=\int_{M} f^{*} \omega_{\phi}$ where $f$ is any section ${ }^{1}$ of $E_{\phi}$. Moreover $\omega(\phi)$ is independent of the choice of section. For example taking $\omega$ to be the $G$-invariant volume form on $X$ we obtain a real number $v(\phi)$ which depends on $\phi$.

When $X$ is even-dimensional the Chern-Gauss-Bonnet theorem implies that $v(\phi)$ may be described as an Euler number, i.e. the self-intersection number of any section, which is a topological invariant of $E_{\phi}$. When $X$ is odd-dimensional this volume invariant is related to a more recent kind of topological invariant (based on bounded cohomology and due to Gromov [3]) and is constant on the connected ${ }^{2}$ components of $\operatorname{Hom}(\pi, G)$.

The volume invariant satisfies an inequality

$$
|v(\phi)| \leqslant \operatorname{volume}(M)
$$

(where volume $(M)=|v(i)|, i: \pi \rightarrow G$ is the identity). For $G=\operatorname{PSL}(2 ; \mathbf{R})$ we recover the famous inequality of Milnor [9] and Wood [17] bounding the Euler number of circle bundles over surfaces admitting flat structures.

Received by the editors August 13, 1981.

1980 Mathematics Subject Classification. Primary 22E40, 57R20, 20H10; Secondary 57S30, $57 \mathrm{R} 32$.

1 Sections exist and are all homotopic since $X$ is contractible.

2 In the "usual" topology, not the Zariski topology.

(C) 1982 American Mathematical Society 0002-9904/82/0000-0143/\$02.00 
Conjecture A. Equality holds in (*) if and only if $\phi$ is an isomorphism of $\pi$ onto a discfete subgroup of $G$.

We state this as a conjecture since we know it presently except for certain $G$ of rank 1. For $G$ compact it is obvious. When $\operatorname{R-rank}((G)>1$ and $\pi \subset G$ is an irreducible ${ }^{3}$ lattice the conjecture may be deduced from Margulis' "superrigidity" theorem [8] (see also [18]) as follows. Margulis proves, under the assumptions on $\pi$ and $G$ above, that unless a homomorphism $\phi: \pi \rightarrow G$ is an isomorphism onto a discrete subgroup (in which case it differs from $i$ by an inner automorphism) the image $\phi(\pi)$ is precompact. In that case there exists $h \in G$ so that $\phi(\pi) \subset h^{-1} K h$ whence $\phi(\pi)$ fixes a point $x \in X$. Letting $f$ be the section of $E_{\phi}$ which is the leaf corresponding to $x$ we obtain $f^{*} \omega_{\phi}=0$ whence $v(\phi)=0$.

For $G$ locally isomorphic to $S O(n, 1)(X=$ hyperbolic $n$-space), Conjecture A may be proved along the lines of Thurston's generalization of Gromov's proof of Mostow rigidity [14, $\S 6.4$, pp. 6.15-6.18] (combined with [5] for the case $n>3)$. See also Gromov's Bourbaki seminar [4].

Now we specialize to the case $G$ is locally isomorphic to $\operatorname{PSL}(2, \mathbf{R})$. For a detailed elementary proof of Conjecture $A$ in this case see [2]. It is interesting to note that the number of values assumed by $v(\phi), \phi \in \operatorname{Hom}(\pi, G)$ is unbounded over all choices $\pi$-sharply contrasting the corollary of Margulis' theorem above.

We will state a formula for the number of connected components of $\operatorname{Hom}(\pi, G)$ in terms of the genus $g$ of the compact Riemann surface $M$. It is a general observation that for $\Gamma$ finitely generated and $G$ an algebraic Lie group the space $\operatorname{Hom}(\Gamma, G)$ is an algebraic variety and has finitely many connected components $^{4}$ - a fact already used in characteristic class discussions (Lusztig, see Sullivan [13] and Gromov [3]).

THEOREM B. The map $v: \operatorname{Hom}(\pi, \operatorname{PSL}(2, \mathbf{R})) \rightarrow \mathbf{R}$ induces an isomorphism of the set of connected components of $\operatorname{Hom}(\pi, \operatorname{PSL}(2, \mathbf{R}))$ onto $\{2 \pi n$ : $n \in \mathbf{Z}$ and $|n| \leqslant 2 g-2\}$.

In particular there are $4 g-3$ components. On the other hand there are only two irreducible components, in the sense of a real algebraic variety. Two of the connected components, corresponding to the maximum and minimum volume and related by changing the orientation of $M$, consist entirely of faithful discrete representations. ${ }^{5}$ Each such component is the space investigated by Weil [15], which is a principal $G$-bundle over the Teichmüller space of $M$.

\footnotetext{
${ }^{3}$ When $\pi \subset G$ is not irreducible the conjecture follows once it is known for the irreducible factors of $\pi$.

4 This is also true if $G$ is semisimple with finite center.

5 In general the subset of $\operatorname{Hom}(\pi, G)$ consisting of faithful discrete representations is closed (by. [7], see also $[11,5.10]$ ) and if $\pi \subset G$ is cocompact also open (by [15], see also $[14,5.1]$.
} 
THEOREM C. Let $G$ be the $n$-fold covering group of PSL $(2, \mathbf{R})$ and $\pi$ the fundamental group of a surface of genus $g$. Then the number of connected components of $\operatorname{Hom}(\pi, G)$ is given by the following formula:

$$
\begin{array}{ll}
2 n^{2 g}+(4 g-4) / n-1, & \text { if } n \mid 2 g-2, \\
2[(2 g-2) / n]+1, & \text { if } n+2 g-2 .
\end{array}
$$

Due to their special significance we briefly mention a few results concerning the components of $\operatorname{Hom}(\pi, G)$ when $\pi$ is a surface group but $G$ is not locally isomorphic to $\operatorname{PSL}(2, \mathbf{R})$. For example $\operatorname{Hom}(\pi, G)$ has two components for $G=$ $P S L(2, \mathrm{C})$ and $S O(3)$ but $\operatorname{Hom}(\pi, G)$ is connected for $G=S U(2)$ (Newstead [10]), $S L(2, \mathrm{C})$, and any 1-connected 3-dimensional Lie group. However if $G$ is not an algebraic Lie group, then $\operatorname{Hom}(\pi, G)$ may have infinitely many components: for the simplest example take $G$ locally isomorphic to the Heisenberg group but not simply connected [2].

Acknowledgment. We wish to thank D. Sullivan and W. Thurston for their inspiration and for generally introducing us to this subject. We are especially grateful to D. Sullivan for his comments on a preliminary version of this paper.

\section{REFERENCES}

1. W. Goldman, Discontinuous groups and the Euler class, Doctoral dissertation, Univ. of California, Berkeley, 1980.

2. - Flat bundles with solvable holonomy. II: Obstruction theory, Proc. Amer. Math. Soc. 83 (1981), 175-178.

3. M. Gromov, Volume and bounded cohomology, Publ. Inst. Hautes Études Sci. Publ. Math. (to appear).

4. - Hyperbolic manifolds according to Thurston and Jprgensen, Séminaire Bourbaki (1979/80), no. 546.

5. U. Haagerup and H. Munkholm, Simplices of maximal volume in hyperbolic space, preprint, Odense University, Denmark.

6. M. Hirsch and W. Thurston, Foliated bundles, flat manifolds, and invariant measures, Ann. of Math. (2) 101 (1975), 369-390. $162-168$

7. D. Kazhdan and G. Margulis, A proof of Selberg's hypothesis, Math. Sb. 75 (1968),

8. G. Margulis, Discrete groups of motions of spaces of nonpositive curvature, Amer. Math. Soc. Transl. (2) 109 (1977), 33-45.

9. J. Milnor, On the existence of a connection with curvature zero, Comment. Math. Helv. 32 (1958), $215-223$.

10. P. Newstead, Topological properties of some spaces of stable bundles, Topology 6 (1967), 241-262.

11. M. S. Raghunathan, Discrete subgroups of Lie groups, Springer-Verlag, Berlin and New York, 1972.

12. A. Selberg, On discontinuous groups in higher dimensional symmetric spaces, Contributions to Function Theory, Tata Institute, Bombay, 1960, pp. 147-164.

13. D. Sullivan, A generalization of Milnor's inequality for affine foliations and affine manifolds, Comment. Math. Helv. 51 (1976), 183-199.

14. W. Thurston, The geometry and topology of 3-manifolds, Princeton Univ. mimeographed notes, 1977-1979. 
15. A. Weil, Discrete subgroups of Lie groups, Ann. of Math. (2) 72 (1960), 369384; 75 (1962), 578-602.

16. H. Whitney, Elementary structure of real algebraic varieties, Ann. of Math. (2) 66 (1957), 545-556.

17. J. Wood, Bundles with totally disconnected structure group, Comment Math. Helv. 51 (1971), 183-199.

18. R. J. Zimmer, Ergodic theory, group representations, and rigidity, preprint, Univ. of Chicago.

DEPARTMENT OF MATHEMATICS, MASSACHUSETTS INSTITUTE OF TECHNOLOGY, CAMBRIDGE, MASSACHUSETTS 02139 